Supporting Information for:

\title{
Methyltrioxorhenium Interactions with Lewis Acid Sites of an Amorphous Silica-Alumina
}

\author{
Anthony W. Moses, ${ }^{1}$ Naseem A. Ramsahye,,${ }^{1}$ Christina Raab, ${ }^{1}$ Heather D. Leifeste, ${ }^{1}$ \\ Swarup Chattopadhyay, ${ }^{1}$ Bradley F. Chmelka, ${ }^{1}$ Juergen Eckert ${ }^{3,4 *}$ and Susannah L. \\ $\operatorname{Scott}^{1,2 *}$ \\ Departments of Chemical Engineering ${ }^{1}$ and Chemistry, ${ }^{2}$ University of California, Santa \\ Barbara CA 93106 \\ ${ }^{3}$ Materials Research Laboratory, University of California, Santa Barbara CA 93106 \\ ${ }^{4}$ Manual Lujan Jr. Neutron Scattering Center, Los Alamos National Laboratory, Los \\ Alamos NM 87545
}

Table of contents:

Figure S1. EXAFS for $\mathrm{CH}_{3} \mathrm{ReO}_{3}$ on silica-alumina, showing curve-fit

S2 without Re- $\mathrm{O}_{\text {support }}$ path.

Table S1. Parameters for EXAFS curvefit for $\mathrm{CH}_{3} \mathrm{ReO}_{3}$ on silica-alumina, without $\mathrm{Re}-\mathrm{O}_{\text {support }}$ path.

Figure S2. EXAFS for $\mathrm{CH}_{3} \mathrm{ReO}_{3}$ on silica-alumina, showing curve-fit to $\quad$ S3 $k^{1}$-weighted data.

Table S2. Parameters for EXAFS curve-fit with $k^{1}$-weighting.

Figure S3. EXAFS for $\mathrm{CH}_{3} \mathrm{ReO}_{3}$ on silica-alumina, showing curve-fit with S4 refined coordination numbers.

Table S3. Parameters for EXAFS curve-fit for $\mathrm{CH}_{3} \mathrm{ReO}_{3}$ on silica-alumina, $\quad \mathrm{S4}$ with refined coordination numbers.

Figure S4. EXAFS for $\mathrm{CH}_{3} \mathrm{ReO}_{3}$ on silica-alumina, showing curve-fit for $\quad$ S5 poly- $\mathrm{CH}_{3} \mathrm{ReO}_{3}$.

Table S4. Parameters for EXAFS curve-fit for $\mathrm{CH}_{3} \mathrm{ReO}_{3}$ on silica-alumina, S5 with poly- $\mathrm{CH}_{3} \mathrm{ReO}_{3}$ model.

Table S5. Cartesian coordinates for aluminosilsesquioxane cube model S6

Table S6. Cartesian coordinates for grafted $\mathrm{CH}_{3} \mathrm{ReO}_{3}$, model I $\quad$ S7

Table S7. Cartesian coordinates for grafted $\mathrm{CH}_{3} \mathrm{ReO}_{3}$, model II $\quad$ S8 
Analysis of EXAFS for $\mathrm{CH}_{3} \mathrm{ReO}_{3}$ on silica-alumina:

Effect of removing the $\mathrm{Re}-\mathrm{O}_{\text {support }}$ path from the Lewis acid-base grafting model
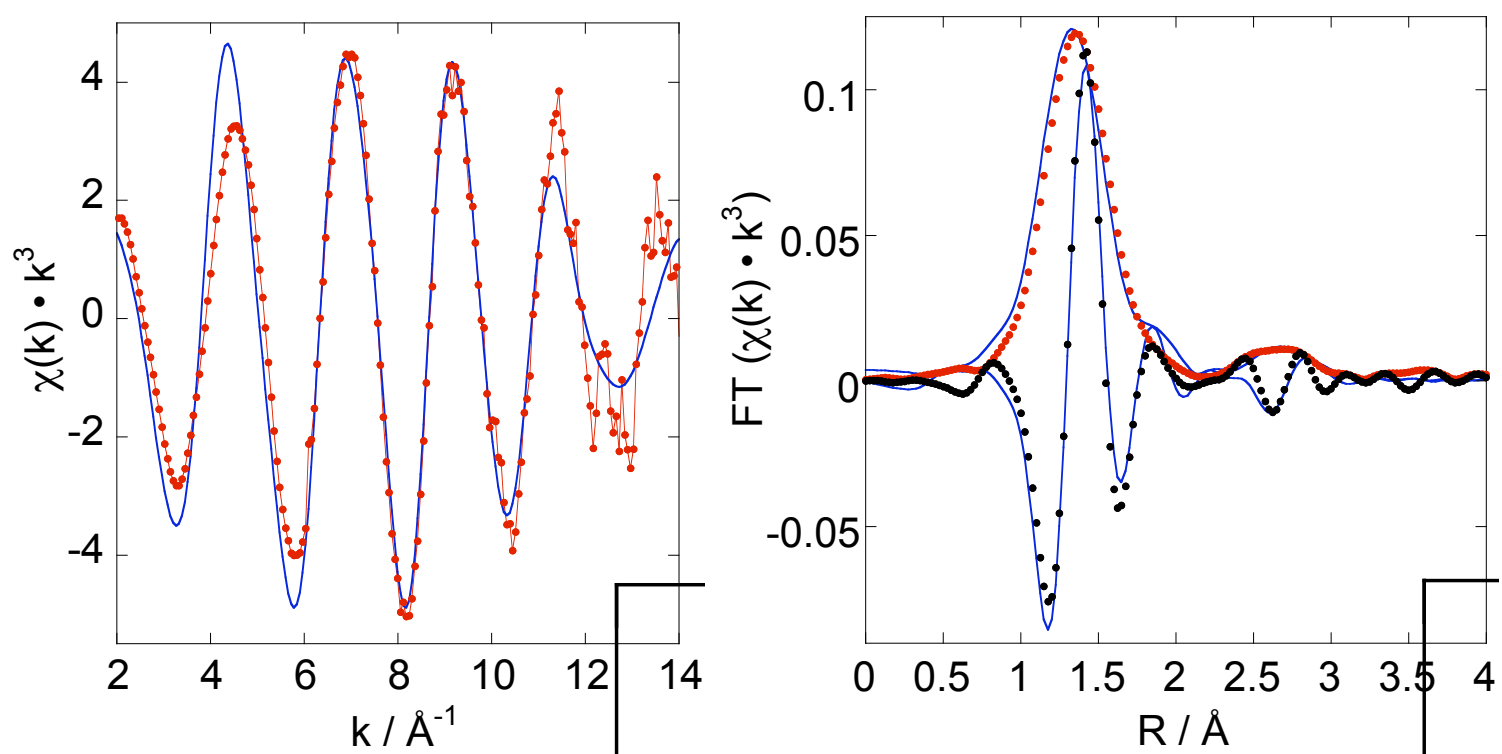

Figure S1. EXAFS data (red and black points, and red line) in $k^{3}$-weighted $k$-space (left) and non-phase-corrected $R$-space (right), for $\mathrm{CH}_{3} \mathrm{ReO}_{3}$ adsorbed on dehydrated silicaalumina (1.3 wt.\% Re). Parameters for the curve-fit (blue lines) are given in Table S1.

Table S1. Parameters from the EXAFS curve-fit for $\mathrm{CH}_{3} \mathrm{ReO}_{3}$ grafted onto silicaalumina, as shown in Figure S1, using the Lewis acid-base adduct model but excluding the $\mathrm{Re}-\mathrm{O}_{\text {support }}$ path. ${ }^{\mathrm{a}}$ Note the physically unreasonable value of the Debye-Waller factor $\sigma^{2}$ for the $\operatorname{Re}=O(N=2)$ path in the first coordination sphere of $R e$.

\begin{tabular}{lccc}
\hline path & $N^{\mathrm{b}}$ & $\mathrm{R}(\AA)$ & $\sigma^{2}\left(\AA^{2}\right)$ \\
\hline $\mathrm{Re}=\mathrm{O}$ & 2 & 1.69 & 0.0010 \\
$\mathrm{Re}=\mathrm{O}$ & 1 & 1.80 & 0.0004 \\
$\mathrm{Re}-\mathrm{C}$ & 1 & 2.11 & 0.0009 \\
$\mathrm{Re}-\mathrm{Al}$ & 1 & 3.07 & 0.0068 \\
\hline
\end{tabular}

${ }^{a}$ Inner potential energies $\left(\Delta E_{0}\right)$ and the global amplitude reduction factor $\left(S_{0}{ }^{2}\right)$ were fixed at $0.0 \mathrm{eV}$ and 1.0, respectively. Residual $=12.7$.

${ }^{\mathrm{b}}$ Not refined. 


\section{Analysis of EXAFS for $\mathrm{CH}_{3} \mathrm{ReO}_{3}$ on silica-alumina: Effect of $\boldsymbol{k}^{1}$-weighting}
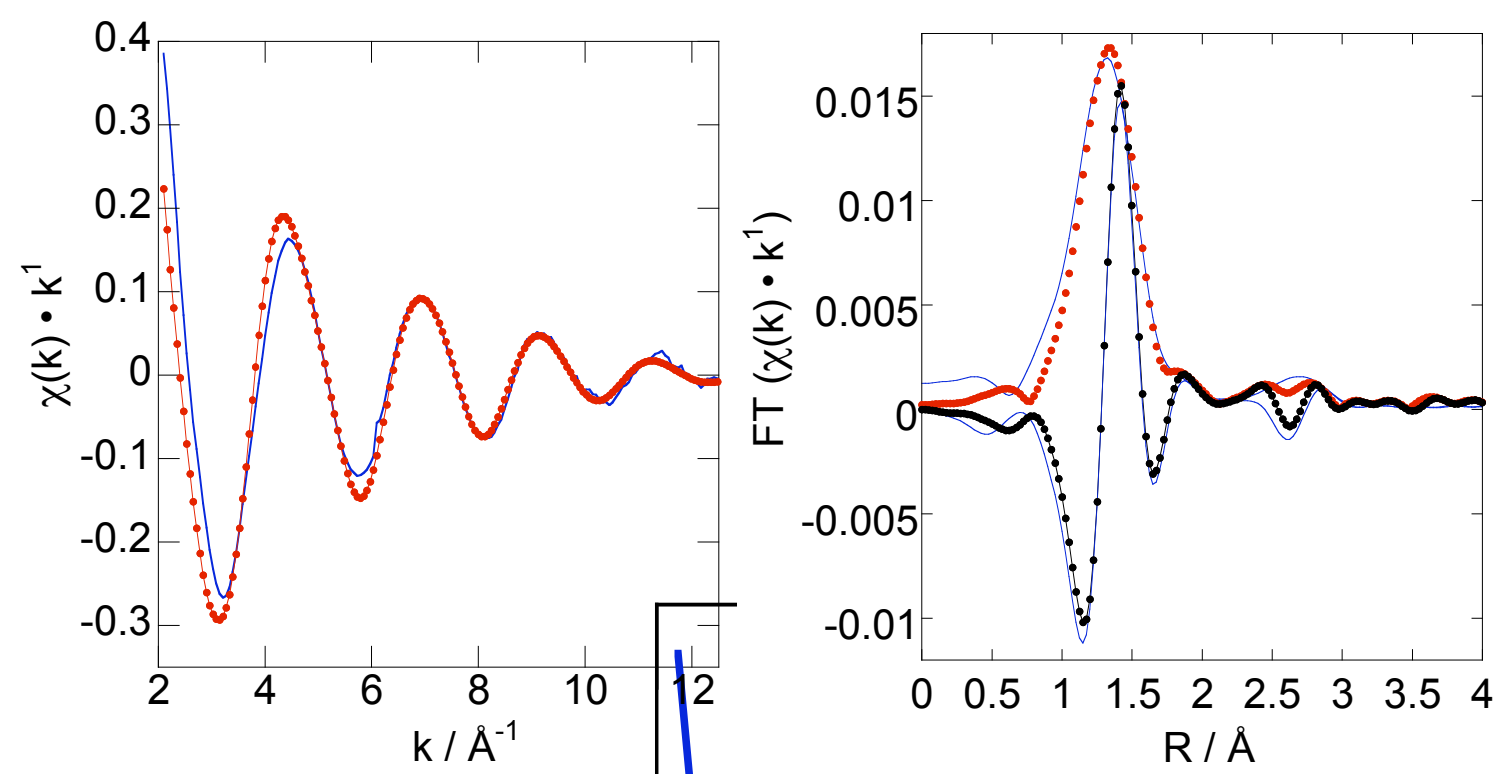

Figure S2. EXAFS data (red and black points and red line) in $k^{1}$-weighted $k$-space (left) and non-phase-corrected $R$-space (right), for $\mathrm{CH}_{3} \mathrm{ReO}_{3}$ adsorbed on dehydrated silicaalumina ( 1.3 wt.\% Re). Parameters for the curve-fit (blue lines) are given in Table S2.

Table S2. Parameters from the EXAFS curve-fit for $\mathrm{CH}_{3} \mathrm{ReO}_{3}$ grafted onto silicaalumina, as shown in Figure S2, for $k^{1}$-weighting. ${ }^{a}$ Note the increased values of the Debye-Waller factors $\sigma^{2}$ for Re-O $O_{\text {support }}$ and Re-Al, relative to the fit in $k^{3}$-weighting (see text-Figure 7).

\begin{tabular}{lccc}
\hline path & $\mathrm{N}^{\mathrm{b}}$ & $\mathrm{R}(\AA)$ & $\sigma^{2}\left(\AA^{2}\right)$ \\
\hline $\mathrm{Re}=\mathrm{O}$ & 2 & 1.70 & 0.0017 \\
$\mathrm{Re}=\mathrm{O}$ & 1 & 1.77 & 0.0020 \\
$\mathrm{Re}-\mathrm{C}$ & 1 & 2.10 & 0.0014 \\
$\mathrm{Re}-\mathrm{O}_{\text {support }}$ & 1 & 2.14 & 0.0072 \\
$\mathrm{Re}-\mathrm{Al}$ & 1 & 3.11 & 0.0107 \\
\hline
\end{tabular}

${ }^{\mathrm{a}}$ Inner potential energies $\left(\Delta \mathrm{E}_{0}\right)$ and the global amplitude reduction factor $\left(\mathrm{S}_{0}{ }^{2}\right)$ were fixed at $0.0 \mathrm{eV}$ and 1.0, respectively. Residual $=11.7$.

${ }^{\mathrm{b}}$ Not refined. 
Analysis of EXAFS for $\mathrm{CH}_{3} \mathrm{ReO}_{3}$ on silica-alumina:

Effect of refining the coordination numbers in the Lewis acid-base grafting model
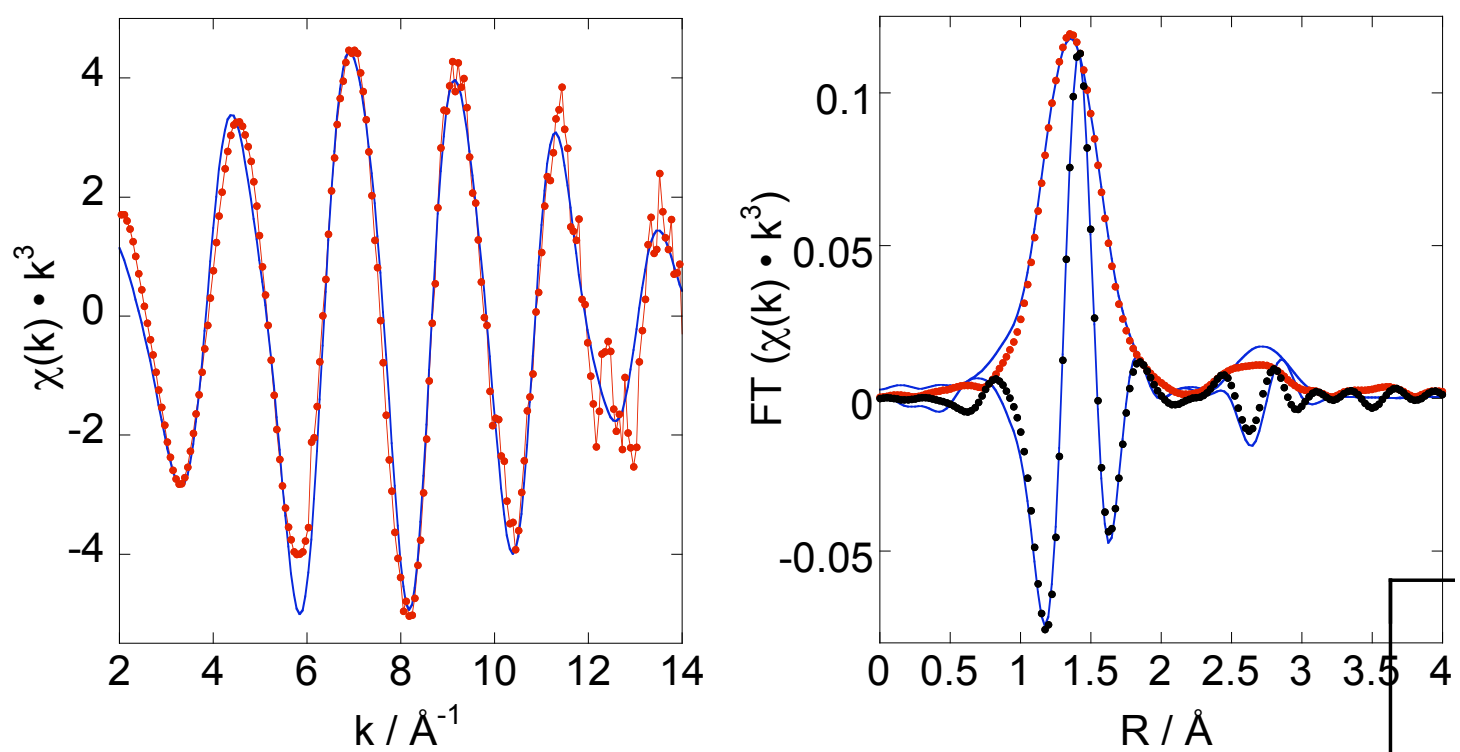

Figure S3. EXAFS data (red and black points and red line) in $k^{3}$-weighted $k$-space (left) and non-phase-corrected $R$-space (right), for $\mathrm{CH}_{3} \mathrm{ReO}_{3}$ adsorbed on dehydrated silicaalumina (1.3 wt.\% Re). Parameters for the curve-fit (blue lines) are given in Table S3.

Table S3. Parameters from the EXAFS curve-fit for $\mathrm{CH}_{3} \mathrm{ReO}_{3}$ grafted onto silicaalumina, as shown in Figure S3, for the full Lewis acid-base grafting model, allowing coordination numbers to refine freely. ${ }^{\mathrm{a}}$

\begin{tabular}{lccc}
\hline path & $\mathrm{N}$ & $\mathrm{R}(\AA)$ & $\sigma^{2}\left(\AA^{2}\right)$ \\
\hline $\mathrm{Re}=\mathrm{O}$ & 1.98 & 1.69 & 0.0016 \\
$\mathrm{Re}=\mathrm{O}$ & 0.85 & 1.77 & 0.0009 \\
$\mathrm{Re}-\mathrm{C}$ & 0.87 & 2.08 & 0.0010 \\
$\mathrm{Re}-\mathrm{O}_{\text {support }}$ & 1.08 & 2.13 & 0.0093 \\
$\mathrm{Re}-\mathrm{Al}$ & 0.89 & 3.08 & 0.0039 \\
\hline
\end{tabular}

${ }^{a}$ Inner potential energies $\left(\Delta E_{0}\right)$ and the global amplitude reduction factor $\left(S_{0}{ }^{2}\right)$ were fixed at $0.0 \mathrm{eV}$ and 1.0 , respectively. Residual $=10.9$. 


\section{Analysis of EXAFS for $\mathrm{CH}_{3} \mathrm{ReO}_{3}$ on silica-alumina: \\ Curve-fit to poly- $\mathrm{CH}_{3} \mathrm{ReO}_{3}$ model}
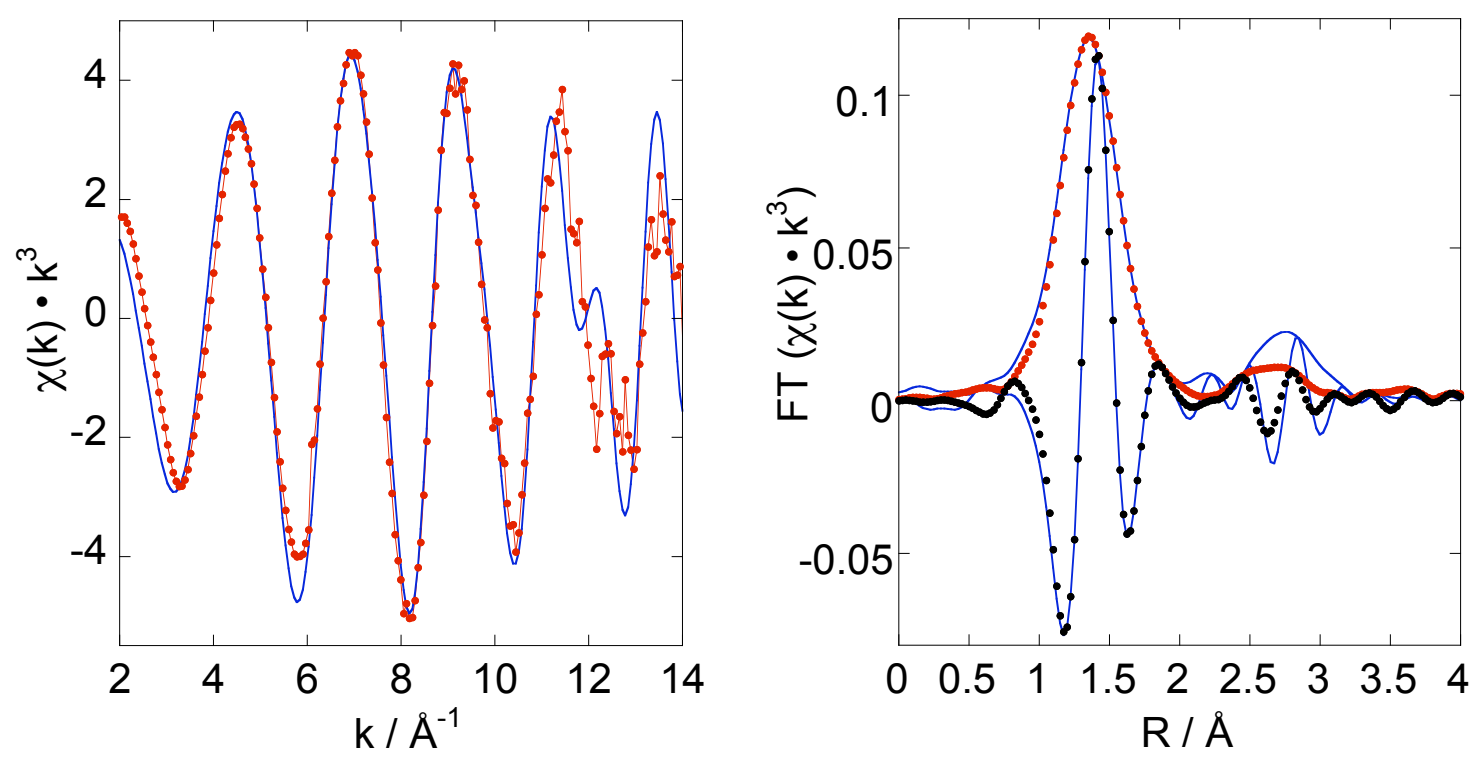

Figure S4. EXAFS data in $k^{3}$-weighted $k$-space (left, red points) and non-phase-corrected $R$-space (right, imaginary component as black points and FT magnitude as red points), for $\mathrm{CH}_{3} \mathrm{ReO}_{3}$ adsorbed on dehydrated silica-alumina (1.3 wt.\% Re). Parameters for the curve-fit (blue lines) are given in Table S2.

Table S4. Parameters from the EXAFS curve-fit for $\mathrm{CH}_{3} \mathrm{ReO}_{3}$ grafted onto silicaalumina, as shown in Figure $\mathrm{S} 2$, fitted to a model of the structure of poly- $\mathrm{CH}_{3} \mathrm{ReO}_{3}$ (corner-sharing $\mathrm{ReO}_{5} \mathrm{CH}_{3}$ octahedra; W. A. Herrmann et al., J. Am. Chem. Soc. 1995, $117,3231-43){ }^{\text {a }}$ Note the physically unreasonable $R e=O$ path length and Debye-Waller factor $\sigma^{2}$ for the Re-Re path.

\begin{tabular}{lccc}
\hline path & $N^{b}$ & $\mathrm{R}(\AA)$ & $\sigma^{2}\left(\AA^{2}\right)$ \\
\hline $\mathrm{Re}=\mathrm{O}$ & 1 & 1.44 & 0.0086 \\
$\mathrm{Re}-\mathrm{O}$ & 4 & 1.71 & 0.0053 \\
$\mathrm{Re}-\mathrm{C}$ & 1 & 2.09 & 0.0015 \\
$\mathrm{Re}-\mathrm{Re}$ & 4 & 2.92 & 0.0197 \\
\hline
\end{tabular}

${ }^{a}$ Inner potential energies $\left(\Delta E_{0}\right)$ and the global amplitude reduction factor $\left(S_{0}{ }^{2}\right)$ were fixed at $0.0 \mathrm{eV}$ and 1.0, respectively. Residual $=11.2$.

${ }^{\mathrm{b}}$ Not refined. 
Table S5. Cartesian coordinates for aluminosilsesquioxane cube model

$\begin{array}{lrrc}\mathrm{H} & 2.761914 & 4.319090 & 1.279784 \\ \mathrm{H} & 3.238209 & -2.796102 & -1.138068 \\ \mathrm{H} & -3.487413 & -1.515772 & -1.267188 \\ \mathrm{H} & 3.054930 & 5.702690 & -0.677835 \\ \mathrm{H} & 4.598083 & 0.426846 & 2.457331 \\ \mathrm{H} & 0.276164 & -0.278054 & -3.980983 \\ \mathrm{H} & -0.547463 & -4.196236 & 1.522663 \\ \mathrm{H} & 0.113198 & -0.377097 & 4.457716 \\ \mathrm{H} & -2.917116 & 2.188289 & 1.780014 \\ \mathrm{O} & -0.809633 & 2.378492 & 0.347025 \\ \mathrm{O} & -1.551075 & -2.290177 & 0.177384 \\ \mathrm{O} & 1.314664 & 3.934573 & -0.907082 \\ \mathrm{O} & 2.068473 & 1.796186 & 0.439682 \\ \mathrm{O} & -0.131282 & -1.853830 & 2.409660 \\ \mathrm{O} & 0.543511 & 1.353823 & -2.032412 \\ \mathrm{O} & 4.114799 & 1.037455 & 1.893888 \\ \mathrm{O} & 1.086670 & -2.764014 & 0.207098 \\ \mathrm{O} & 2.862966 & -0.767359 & 0.332972 \\ \mathrm{O} & -1.179003 & -0.704677 & -1.946559 \\ \mathrm{O} & -0.970901 & 0.689288 & 2.421213 \\ \mathrm{O} & 1.448366 & -1.169913 & -1.915205 \\ \mathrm{O} & 1.655234 & 0.135774 & 2.513521 \\ \mathrm{O} & -2.432137 & 0.237964 & 0.234202 \\ \mathrm{Si} & 0.275027 & -0.175180 & -2.514794 \\ \mathrm{Si} & -1.806761 & 1.419455 & 1.200032 \\ \mathrm{Si} & -0.295137 & -2.817428 & 1.090367 \\ \mathrm{Si} & -2.199533 & -1.073284 & -0.720355 \\ \mathrm{Si} & 2.669417 & 0.555200 & 1.289775 \\ \mathrm{Si} & 2.187644 & -1.895655 & -0.648852 \\ \mathrm{Si} & 0.160424 & -0.344344 & 2.991213 \\ \mathrm{Al} & 0.676038 & 2.196333 & -0.524742 \\ \mathrm{Si} & 2.870410 & 4.305259 & -0.188178 \\ \mathrm{H} & 3.914023 & 3.447047 & -0.772421 \\ \mathrm{Si} & 0.417124 & 5.043444 & -1.880030 \\ \mathrm{H} & 0.117855 & 6.232281 & -1.049903 \\ \mathrm{H} & 1.256672 & 5.401849 & -3.045304 \\ \mathrm{H} & -0.803362 & 4.312782 & -2.272630\end{array}$


Table S6. Cartesian coordinates for $\mathrm{CH}_{3} \mathrm{ReO}_{3}$ grafted to an aluminosilsesquioxane cube with four-coordinate $\mathrm{Al}$, model I

$\begin{array}{lrrc}\mathrm{C} & -1.956289 & 3.759057 & 2.841259 \\ \mathrm{H} & 4.340286 & 0.934363 & -0.126759 \\ \mathrm{H} & -0.716544 & -1.756818 & 3.579016 \\ \mathrm{H} & -2.444894 & 4.538691 & 3.429243 \\ \mathrm{H} & 2.634741 & -3.550526 & 0.572025 \\ \mathrm{H} & -2.665748 & 2.946351 & 2.635490 \\ \mathrm{H} & -1.089385 & 3.352202 & 3.367973 \\ \mathrm{H} & -0.427676 & -3.003481 & -3.151349 \\ \mathrm{H} & 1.778036 & 3.403181 & 2.873573 \\ \mathrm{H} & -3.779647 & -1.341402 & -0.147810 \\ \mathrm{H} & 1.372364 & 1.398048 & -3.989191 \\ \mathrm{O} & -2.340788 & 0.747570 & -0.427353 \\ \mathrm{O} & -1.932734 & 3.689144 & -0.501723 \\ \mathrm{O} & 0.790234 & -2.130379 & 1.572522 \\ \mathrm{O} & -0.406434 & 2.449589 & 0.953376 \\ \mathrm{O} & 0.203651 & 5.097415 & 1.093382 \\ \mathrm{O} & 0.180852 & 0.321718 & 2.451610 \\ \mathrm{O} & 2.397054 & 0.809756 & -1.751086 \\ \mathrm{O} & -1.553720 & -1.677538 & -1.307573 \\ \mathrm{O} & -1.724258 & -1.194385 & 1.320379 \\ \mathrm{O} & 0.412846 & -0.634872 & -2.825059 \\ \mathrm{O} & 0.956704 & -2.590266 & -1.070458 \\ \mathrm{O} & 1.216729 & 2.648418 & 3.096313 \\ \mathrm{O} & 2.795644 & -1.055946 & 0.155473 \\ \mathrm{O} & -0.065726 & 1.856806 & -1.924776 \\ \mathrm{O} & -2.363844 & 5.837479 & 1.061972 \\ \mathrm{O} & 2.047668 & 1.422502 & 0.838352 \\ \mathrm{Re} & -1.361582 & 4.497777 & 0.987762 \\ \mathrm{Si} & 1.812559 & -2.365242 & 0.307529 \\ \mathrm{Si} & 0.789499 & 1.693297 & 1.833461 \\ \mathrm{Si} & -2.393701 & -0.854004 & -0.155213 \\ \mathrm{Si} & 2.933592 & 0.531544 & -0.233100 \\ \mathrm{Si} & -0.165969 & -1.994978 & -2.118326 \\ \mathrm{Si} & 1.013090 & 0.890965 & -2.658379 \\ \mathrm{Si} & -0.385707 & -1.215379 & 2.256807 \\ \mathrm{Al} & -1.185901 & 2.007347 & -0.634364 \\ & & & \end{array}$


Table S7. Cartesian coordinates for $\mathrm{CH}_{3} \mathrm{ReO}_{3}$ grafted to a siloxane $\left(\mathrm{H}_{3} \mathrm{SiOSiH}_{3}\right)$ capped aluminosilsesquioxane cube with five-coordinate $\mathrm{Al}$, model II

$\begin{array}{lccc}\mathrm{Al} & 1.832134 & 1.585133 & -0.272832 \\ \mathrm{C} & 0.494545 & 4.254351 & 2.474363 \\ \mathrm{H} & 3.258494 & -1.611850 & 3.633160 \\ \mathrm{H} & 1.231932 & 3.569631 & 2.915226 \\ \mathrm{H} & -2.157498 & 3.112701 & 0.939940 \\ \mathrm{H} & 1.804288 & -4.079187 & -0.398020 \\ \mathrm{H} & 4.630532 & -0.789915 & 0.211361 \\ \mathrm{H} & -0.497060 & 3.816806 & 2.600472 \\ \mathrm{H} & 5.035055 & 1.410692 & 1.293146 \\ \mathrm{H} & 6.178994 & 0.783098 & -0.740410 \\ \mathrm{H} & 0.552470 & 5.225527 & 2.969937 \\ \mathrm{H} & 4.352844 & 0.731248 & -3.435102 \\ \mathrm{H} & -1.443427 & -0.302452 & 4.275335 \\ \mathrm{H} & -3.444651 & -0.016928 & -2.209054 \\ \mathrm{H} & -2.790757 & -3.614195 & 1.026424 \\ \mathrm{H} & 5.316263 & 2.765853 & -2.522426 \\ \mathrm{H} & 1.145887 & -0.701072 & -3.749192 \\ \mathrm{H} & 2.940505 & 2.691311 & -3.062433 \\ \mathrm{O} & 1.920614 & 5.817565 & 0.777345 \\ \mathrm{O} & -2.542263 & -1.528664 & -0.390700 \\ \mathrm{O} & 2.275922 & 3.399189 & -0.308396 \\ \mathrm{O} & 3.749158 & 1.379841 & -0.995912 \\ \mathrm{O} & 1.866338 & -2.036570 & 1.091657 \\ \mathrm{O} & -1.646881 & -1.647642 & 2.139943 \\ \mathrm{O} & 1.171067 & 0.803262 & -1.679195 \\ \mathrm{O} & 2.347587 & 0.616182 & 1.093024 \\ \mathrm{O} & 3.323153 & -0.867334 & 3.022100 \\ \mathrm{O} & -0.441009 & -3.092112 & 0.224580 \\ \mathrm{O} & -1.875785 & 1.058964 & -0.521194 \\ \mathrm{O} & -1.386379 & 1.026125 & 2.122560 \\ \mathrm{O} & -0.986807 & -0.629365 & -2.400917 \\ \mathrm{O} & 0.247469 & 2.402618 & 0.477116 \\ \mathrm{O} & 1.214879 & -1.883556 & -1.517380 \\ \mathrm{O} & -0.290693 & 4.936487 & -0.449866 \\ \mathrm{O} & 0.672855 & -0.505796 & 2.901896 \\ \mathrm{Re} & 1.028396 & 4.429784 & 0.455392 \\ \mathrm{Si} & 2.045306 & -0.679234 & 2.005477 \\ \mathrm{Si} & -0.956042 & -0.363954 & 2.892161 \\ \mathrm{Si} & 1.131064 & -2.795972 & -0.161171 \\ \mathrm{Si} & -1.324231 & 1.918095 & 0.746784 \\ \mathrm{Si} & 4.100308 & 1.917136 & -2.579907 \\ \mathrm{Si} & -2.240506 & -0.274505 & -1.410857 \\ \mathrm{Si} & 0.668112 & -0.571486 & -2.363195 \\ \mathrm{Si} & 4.912101 & 0.647926 & 0.036292 \\ \mathrm{Si} & -1.875285 & -2.500738 & 0.753475\end{array}$

